\title{
The Complete Makeover of Cloud Computing in the Next Decade, Will You Survive?: A Prophetic Approach
}

\author{
Duncan WAGA ${ }^{1, *}$, Kefa RABAH ${ }^{2}$ \\ ${ }^{1}$ Jaramogi Oginga Odinga University of Science and Technology, Kenya \\ ${ }^{2}$ Kabarak University, Kenya \\ *Corresponding Author: wagadun@gmail.com
}

Copyright (C) 2013 Horizon Research Publishing All rights reserved.

\begin{abstract}
Despite the young age of Cloud Computing (CC), there is already tremendous pressure to metamorph its structure due to the dynamic nature of technology in the virtualization environment. Cloud providers are supplying users with varied flavors of CC while the latter haphazardly implements their systems to stay an edge over their competitors. Solution to the numerous technical and otherwise challenges affecting $\mathrm{CC}$ currently will compel a total overhaul of the $\mathrm{CC}$ architecture. The next decade will doubtlessly see a completely different $\mathrm{CC}$ with possible compatibility issues to current user systems. This paper presents the most probable changes that will hit the CC and outlines what end users should brace themselves to. The year 2020 is used as a reference point and federated datacenters behaving like breathing organisms with changing states are discussed. Views from a cross section of Industry is given and recommendations are suggested to users who are currently planning to take up CC.
\end{abstract}

Keywords Cloud Computing, Virtualization; Cloud Providers, Cloud Flavor, Federated Datacenters

\section{Introduction}

CC is hardly six years old Kamthania (2009) and is already undergoing a lot of Technological pressure as it finds its space in the virtualization environment. As the market forces influence $\mathrm{CC}$ adoption many systems are finding their way into different flavors of $\mathrm{CC}$ which are as a result of various technologies. As the latter is improved for better performance and cost, the initial form of $\mathrm{CC}$ is bound to change. These changes may be so profound that the resident systems may no longer achieve their intended goals. This is a matter of grave concern especially coming at a time huge investments are being committed to these systems, a phenomenon that is likely like bound to cause a lot of panic in the already confused cloud market.

In the next decade several changes will have occurred either in the market, technology or user profiles. This paper outlines these changes and gives recommendations that users should pursue to alleviate or prepare for such happening. So far we know there will be larger clouds which will link to others says Jack Clark from cloud watch and most services that businesses consume will sit on top of these clouds. Software will be much, much larger. The European Commission has increased its funding from $€ 630 \mathrm{~m}$ to $€ 1.2 \mathrm{bn}$ advanced to the Digital Agenda investment in high-performance computing aiming to reverse Europe's decline in the field so the region can build an exascale system before the end of the decade. This will be geared towards investment on super computing, research and training between now and the year 2020 which will obviously affect cloud computing since it targets the data centers. According to Cisco, these "things" of the real world shall seamlessly integrate into the virtual world, enabling anytime, anywhere connectivity. In 2010, the number of everyday physical objects and devices connected to the Internet was around 12.5 billion. They forecast that this figure is expected to double to 25 billion in 2015 as the number of more smart devices per person increases, and to a further 50 billion by 2020 as Figure 1 stipulates.

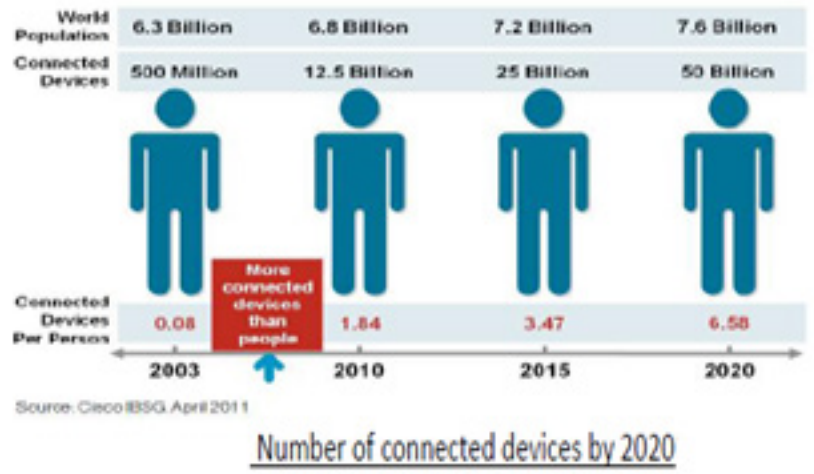

Figure 1. Number of connected devices by 2020

\section{Literature Review}

Kamthania et al (2009) will involve assembling and 
connecting various existing online services. Companies like Google and Microsoft have taken this a step further already by creating many tools that are becoming increasingly more integrated. The next step would be to fully move the operating system online, making hardware- whether a desktop, laptop, ultra portable, phone, etc. - simply a terminal to access the cloud of which some companies are already experimenting with. The Blue cloud, an IBM initiative to be based on open standards and open source under over 200 based internet scale worldwide targeting clients interested in $\mathrm{CC}$ infrastructures quickly and easily Buyya et al (2010). Federated clouds, an amalgamation of several clouds able to predict the distribution of its users and automatically balancing their respective loads will be common place and users who strictly have applications in single clouds will be forced to re draft their policies.

Chang (2010) discusses that the next decade will have CC architectures for creating market-oriented Clouds and computing atmosphere by leveraging technologies such as virtual machines, provides thoughts on market-based resource management strategies that encompass both customer-driven service management and computational risk management to sustain SLA-oriented resource allocation Abas et al (2010) in his work with educational CC innovation predicts that not only would educators have to cope with new technologies, they also will have to deal with a new generation of learners; learners of different learning attitudes and styles. The Generation Y and mobile technology, games technology and 3D virtual worlds will greatly influence the operation of CC and applications there in. In their study of computing with molecules Reed et al (2000) they argued that computers will be very powerful and fast and may have possibilities create artificial "brains" that have intellectual capabilities comparable or even superior to those of human beings. This is because of the breakthrough of making computer circuits using molecules as opposed to the semi conductor based solid state micro electronics which will lead to circuitry dense and complex enough to give rise to true cognitive abilities.

\section{Molecular Logic Circuits}

Balzani (2002) researched on evolution aspects of miniaturization which has been an essential ingredient in the outstanding progress of information technology over the past fifty years. The next, perhaps ultimate, limit of miniaturization is that of molecules, which are the smallest entities with definite size, shape, and properties he said. Recently, great effort has been devoted to design and investigate molecular-level systems that are capable of transferring, processing, and storing information in binary form. Some of these nanoscale devices can, in fact, perform logic operations of remarkable complexity. This research-although far from being transferred into technology - is attracting interest, as the nanometer realm seems to be out of reach for the "top-down" techniques currently available to microelectronics industry. Moreover, such studies introduce new concepts in the "old" field of chemistry and stimulate the ingenuity of researchers engaged in the "bottom-up" approach to nanotechnology. Pischel argues that to commit to memory: Whereas combinational logic devices are indifferent with respect to the history of input application, sequential logic is related to a memory function. A proof-of-principle of the sequential logic concept at the molecular level was demonstrated recently with a surface-confined osmium complex which is an important conceptual advance toward molecular information processing. Joachim et al 2000 says the semiconductor industry has seen a remarkable miniaturization trend, driven by many scientific and technological innovations. But if this trend is to continue, and provide ever faster and cheaper computers, the size of microelectronic circuit components will soon need to reach the scale of atoms or molecules-a goal that will require conceptually new device structures. The idea that a few molecules, or even a single molecule, could be embedded between electrodes and perform the basic functions of digital electronics-rectification, amplification and storage - was first put forward in the mid-1970s. The concept is now realized for individual components, but the economic fabrication of complete circuits at the molecular level remains challenging because of the difficulty of connecting molecules to one another. A possible solution to this problem is 'mono-molecular' electronics, in which a single molecule will integrate the elementary functions and interconnections required for computation. Tian 2010 researched on data processing through molecules and says the integration of complex logic functions within individual molecules allows data processing at the molecular level, as demonstrated by recently reported molecular encoder and decoder devices. This research on decision-making molecules has great potential for future applications and more complex computing on a functional unimolecular platform. Raymo 2002 says organic molecules are promising candidates for the realization of future digital processors. Their attractive features are the miniaturized dimensions and the high degree of control on molecular design possible in chemical synthesis. Indeed, nanostructures with engineered properties and specific functions can be assembled relying on the power of organic synthesis. In particular, certain molecules can be designed to switch from one state to another, when addressed with chemical, electrical, or optical stimulations, and to produce a detectable signal in response to these transformations. Binary data can be encoded on the input stimulations and output signals employing logic conventions and assumptions similar to those ruling digital electronics. Thus, binary inputs can be transduced into binary outputs relying on molecular switches. Following these design principles, the three basic logic operations (AND, NOT, and OR) and more complex logic functions (EOR, INH, NOR, XNOR, and XOR) have been reproduced already at the molecular level. Presently, these simple "molecular processors" are far from any practical application. However, these encouraging results demonstrate already that chemical systems can process binary data with designed logic protocols. Further 
fundamental studies on the various facets of this emerging area will reveal if and how molecular switches can become the basic components of future logic devices. After all, chemical computers are available already. We all carry one in our head!

\section{CC Challenges of the Next Decade}

Clark (2012) of cloud watch asserts that in the year 2020 $\mathrm{CC}$ is set to become a standard part of enterprise IT despite facing numerous challenges currently. The low-power, scalable smart clouds of 2020 hold great promise. In particular, operating at cloud scale means there will be more hardware and software failures and dealing with these failures will be an important issue. To get an idea of the type of failures that cloud companies will be forced to deal with, it's helpful to look to supercomputing - an area that uses many of the technologies and methods that eventually make their way into the cloud. "You're scaling up the number of cores, so the number of failures - hardware and system software failures - go up dramatically," says Richard Kenway, head of the Scientific Steering Committee of PRACE - a European scheme that aims to pool the resources of various supercomputers across the region to create a system capable of exascale computation. "I've heard claims it could be an undetected failure every few minutes." This means new software systems will have to be developed to help deal with the likelihood of minute-to-minute failures in the underlying infrastructure. The core failure rate will be compounded by a reduction in memory bandwidth to each core, Kenway says, as he expects the number of cores developed to outpace the rise in bandwidth. This will increase the software issues that programmers face, and could force them to have to think about new ways of developing software.

What separate the cloud from supercomputers are the types of hardware choices that need to be made. With supercomputers, huge emphasis is placed on single-threaded performance, so we can expect these systems to focus on high-end chips e.g. Xeons, while clouds care more about 'dumb' workloads that require less single-threaded performance. This means less capable - but lower-power - chips will start to make inroads into the cloud, hugely increasing the number of chips stewarded by clouds and causing a shift in software development strategies, adds Clark.

New software systems will have to be developed to help deal with the likelihood of minute-to-minute failures in the underlying infrastructure. By 2020, Fujitsu Technology Solution's chief technology officer, Josef Reger, expects the needs of the cloud will favor low-power chips with many cores of course with numerous complications.

Companies will need to standardize infrastructures and bring their application development in line with the chips they use. They will also need to program their cloud operating systems to be much more parallel to deal with the memory crunch. These two issues, combined with the larger scale at which clouds are likely to operate, could cause headaches for developers.

Though new networking technologies - faster interconnects, better on-chip communication, and so on will go some way toward speeding the pace at which updates can ripple through a system, challenges will remain, especially around automating the update process within large applications.

But there's not much point having these huge clouds running off low-power chips if silly mistakes mean you do not get as much efficiency out of them as you could, according to Face book's VP of hardware design and supply chain, Frank Frankovsky. "One of the big orchestration layer challenges that I think is interesting to me or anyone running a large-scale datacenter is not how you build efficient infrastructure, but how do you operate it efficiently?" he says. Frankovsky believes capping and managing power consumption is an area that needs more investment. And while today vendors solve these issues at the server level or rack level, it will take an industry-wide open initiative to solve it for large datacenters.

Face book has embarked on the Open Compute Project, which hopes to standardize the chunks of infrastructure that go into the datacenter, to make life easier for people in charge of maintaining cloud datacenters. Though hardware standardization leads to an increased emphasis on software, which has benefits for management, the proliferation of hardware as clouds grow will mean serviceability will become ever more important. "Because there's tens of thousands of devices, the ability for the technician to identify the faulty device, replace the component and get it back into operation is a really important part of operations at scale," Frankovsky says. Vendors are already working to solve these problems. Some HP servers come with a technology HP has called a "sea of sensors" that lets them self-diagnose problems and specific equipment failures, while Face book has created various software agents that let them reconfigure servers over the network, without having to physically go and manipulate them to modify their BIOS. It seems likely this scheme will have traction. Adrian Cockcroft, Netflix's cloud architect, advocates exactly the same open source hardware approach that Face book has called for. He says when Netflix decided to build its own content distribution network (CDN) to make sure its online video service worked smoothly for users, it designed its own hardware as well.

As with any technology, a lot of the true problems could come in implementation. Even large providers with a wealth of experience can struggle to deal with their scale says Adrian Cockcroft, Netflix. As Bryan Ford, a Yale academic who researches cloud stability, puts it: "Such risks are still extremely challenging, even with one organization, because of the standard 'left hand doesn't know what the right hand is doing' issue." Cloud computing brings with it a whole new set of applications that will sit on multiple tiers of cloud infrastructure, so there will be a need for communication between all the parties involved in any one cloud. This is an 
organizational issue as much as a technological one and some companies are already trying to solve it. Netflix, for instance, has moved to a "no-ops" development strategy where each developer is responsible for their own code and making sure it can deal with failures in any of the other bits of code it talks to. This strikes Cockcroft as the best way to avoid problems of interdependency at scale. "All of our components are designed to assume that the things around them will fail and they have to keep working," he says. "At any point in time there's a fair amount of stuff that's probably broken that customers don't notice because the service routes around [it]. I think probably in two years time you'll see that become relatively mainstream." Related to the problems of communication, both from an organizational and a technical perspective, is the challenge of developing true multi-tenant software applications. As recent outages by Amazon have shown, developing this technology is non-trivial, even for the world's largest public cloud company, and it's going to get more difficult as more organizations consume multiple applications from multiple clouds.

"One of the big orchestration layer challenges is not how you build efficient infrastructure, but how do you operate it efficiently" Frank Frankovsky, Face book. John Manley, who runs HP Lab's automated infrastructure lab, says "the challenge ahead is going to be the production of true multi-tenant software by a software author." This is because as multiple organizations access a single piece of software from one cloud, the developer needs to make sure that data is kept separate, and charge back is being handled effectively. At the end of every session the instance needs to be torn down and returned back to the compute pool, Manley says, which can be tricky if companies are renting software for a large period of time.

Taken together, all the barriers to the cloud can be solved if industry adopts two things: standards and full technical disclosure. Standards will make it easier to manage software and hardware at scale, while full technical disclosure will stop interdependencies causing problems. But enterprise incumbents are going to fight a pitched battle against both of these things. Standards beget commoditization and have the potential to cut into the juicy profit margins that major vendors can charge for their proprietary technology. Combined with this, full technical disclosure of cloud architectures should make it easier for start-ups to enter into the market with technology on a par with incumbents, further disrupting the market. Some vendors have acknowledged this threat; HP and Huawei have joined Face book's Open Compute Project, though continue to try and carve off bits of the open technology and use it for their proprietary endeavors. If the past is anything to go on, between now and 2020, we can look forward to a raft of conflicting standards from interested parties - and regular quibbling over exactly how open any one vendor needs to be.

\section{New Look CC}

The kind of CC likely to show up come the year 2020 may exhibit close to the following aspects as displayed in Figure 2.

\section{Convergence of Technologies}

Figure 3 clearly shows how Transistor evolution is leading to different processor powers and hence more agile CCs.

Some new approaches are taking shape for datacenter design. Fabric-based infrastructure would benefit from converging SAN and LAN fabrics to allow pools of servers to communicate freely to pools of storage. 50 percent of today's 21-year-olds have produced content for the web. 70 percent of four-year-olds have used a computer while the average teenager writes 2,282 text messages on their cell phone per month. This year alone, Google averaged 31 billion searches per month, compared 2.6 billion searches per month in 2007.More video has been uploaded to YouTube in the last two months than the three major US networks (ABC, NBC, CBS) have aired since 1948. Wikipedia averages 4300 new articles per day, and now has over 13 million articles. This year, Face book reached 500 million users. If it were a country, it would be ranked third. Twitter would be ranked 7 th, with $69 \%$ of their growth being from people $32-50$ years old. In 1997, a GB of flash memory cost nearly $\$ 8000$ to manufacture; today it is only $\$ 1.25$ instead. The computer in today's cell phone is million times cheaper, and thousand times more powerful, than a single computer installed at MIT back in 1965. In 25 years, the compute capacity of today's cell phones could fit inside a blood cell. Summarily, the CC of the next decade is much more likely to exhibit these kinds of unequalled attributes. Since it is a technology that is influenced by a convergence of trends, the following table outlines the possible aspects which will help reshape CC. Below are diagrammatic representation of the various convergences.

\section{Trends of Technologies}

Technology facets show up periodically as shown in Figure 4.However disruptive technologies can interfere with any meaningful prediction since they often change the trends.

Over the years performance of computers have exponentially gone up as the years progress. Figure 5 may help us predict supercomputer performance in the next decade

Users tend to prefer the SaaS followed by the IaaS, PaaS and BPaaS and the flavors get more popular as the decade approaches as shown in Figure 6. 


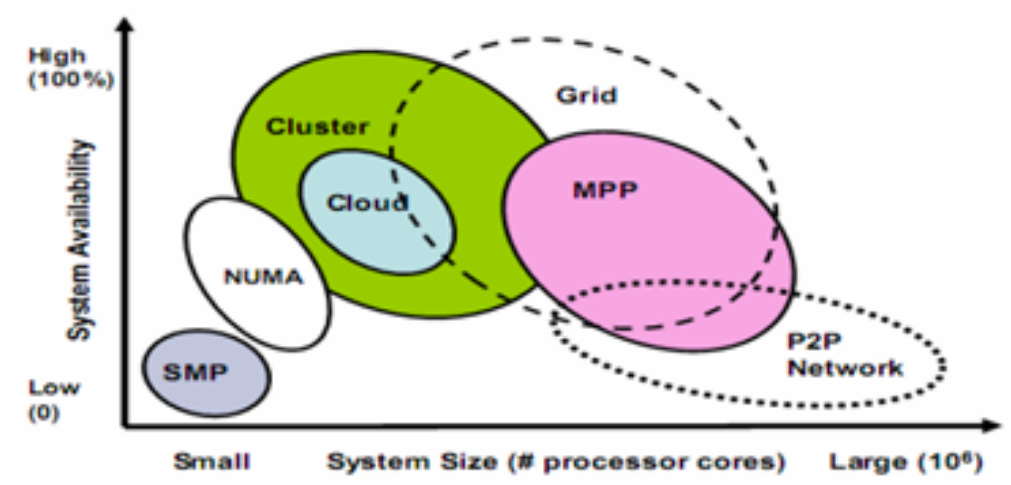

Estimated effects on the system availability by the size of clusters, MPP, Grids, P2P file-sharing networks, and computing clouds. (The estimate is based on reported experiences in

hardware, OS, storage, network, and packaging technologies in available system configurations in 2010.)

Figure 2. Factors that would influence $\mathrm{CC}$ in the coming decade

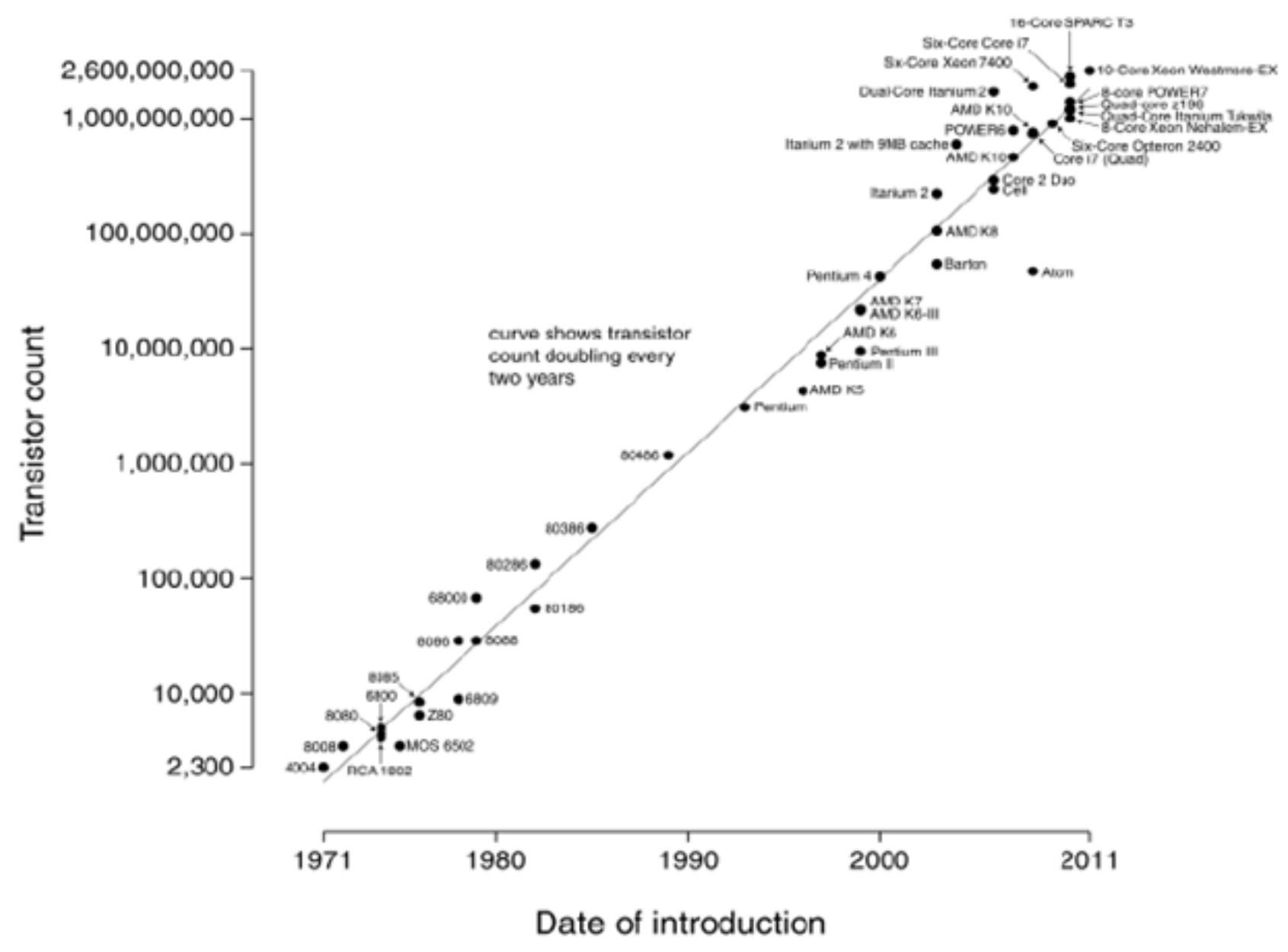

Figure 3. Data of introduction 


\section{ASSUMED TIMELINES FOR THE FIFTEEN TRENDS}

These timelines were assumed for the trends and when they might emerge and evolve, with the caveat that new and potentially disruptive technologies could change everything.

\begin{tabular}{|c|c|c|c|c|c|}
\hline & $\begin{array}{c}0-3 \\
\text { years }\end{array}$ & $\begin{array}{c}3-5 \\
\text { years }\end{array}$ & $\begin{array}{c}5-7 \\
\text { years }\end{array}$ & $\begin{array}{c}7+ \\
\text { years }\end{array}$ & $\begin{array}{c}\text { Group's } \\
\text { top } 4 \text { for } \\
2013\end{array}$ \\
\hline 1. Mobility & $\gamma$ & 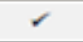 & $\gamma$ & $\gamma$ & $\gamma$ \\
\hline 2. Natural user interface & & $\gamma$ & $\gamma$ & $\gamma$ & \\
\hline 3. Personalization & $\gamma$ & - & $\gamma$ & $\gamma$ & 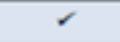 \\
\hline 4. Socially-enabled enterprise/consumerization & & $\gamma$ & $\gamma$ & $r$ & \\
\hline 5. Visualization management & & & $\gamma$ & $\gamma$ & \\
\hline 6. Internet of things & $\gamma$ & $\gamma$ & $\gamma$ & $\gamma$ & $\gamma$ \\
\hline 7. Lean agile process & $\checkmark$ & $\checkmark$ & & $\checkmark$ & \\
\hline 8. Business transformation & $\checkmark$ & $\checkmark$ & & & \\
\hline 9. Robotics & $\checkmark$ & $\checkmark$ & $\checkmark$ & $\checkmark$ & \\
\hline 10. Artificial Intelligence (A) & & $\checkmark$ & $\checkmark$ & & \\
\hline 11. Augmented Reality (AR) & & $\checkmark$ & $\checkmark$ & $\checkmark$ & \\
\hline 12. Three Dimensional ( 3-D) Printing & $\checkmark$ & $\checkmark$ & & & \\
\hline 13. Telepresence & $\checkmark$ & $\checkmark$ & & & \\
\hline 14. Security & $\checkmark$ & $\checkmark$ & $\checkmark$ & $\checkmark$ & $\checkmark$ \\
\hline 15. Power generation/consumption/managem & & & $\checkmark$ & & \\
\hline
\end{tabular}

OCopyright North Central Texas InterLink, Inc. All rights reserved

Figure 4. Assumed timelines for the fifteen trends

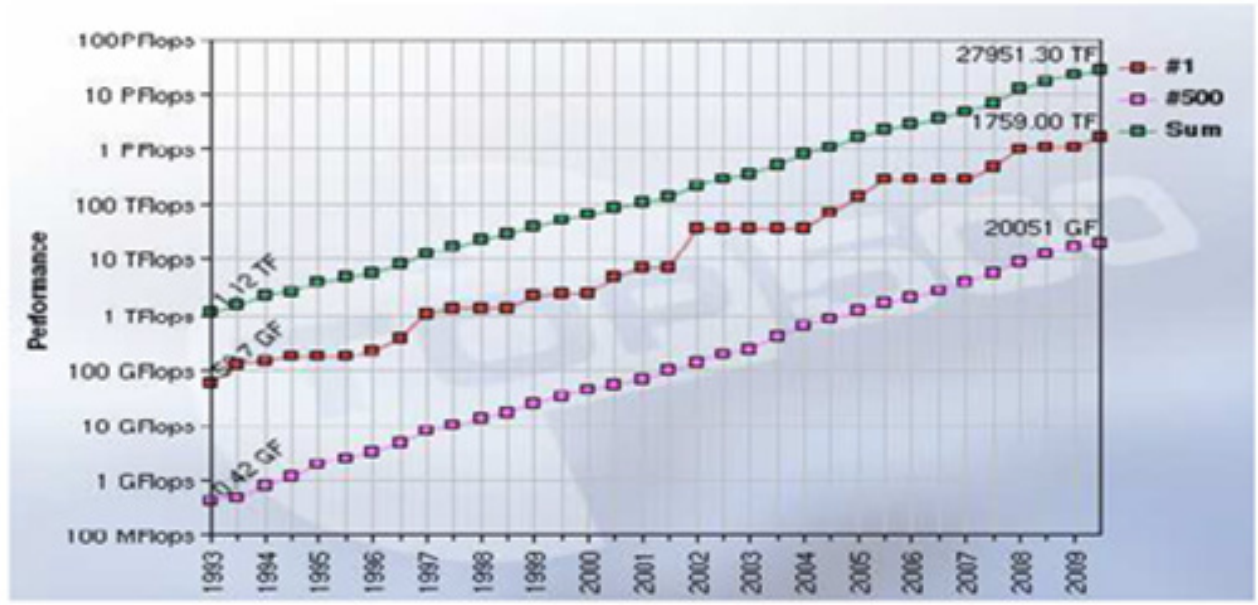

The Top-500 supercomputer performance (Courtesy of Top 500 Organization, 2009)

Figure 5. The Top-500 supercomputer performance (Courtesy of top 500 Organization,2009) 


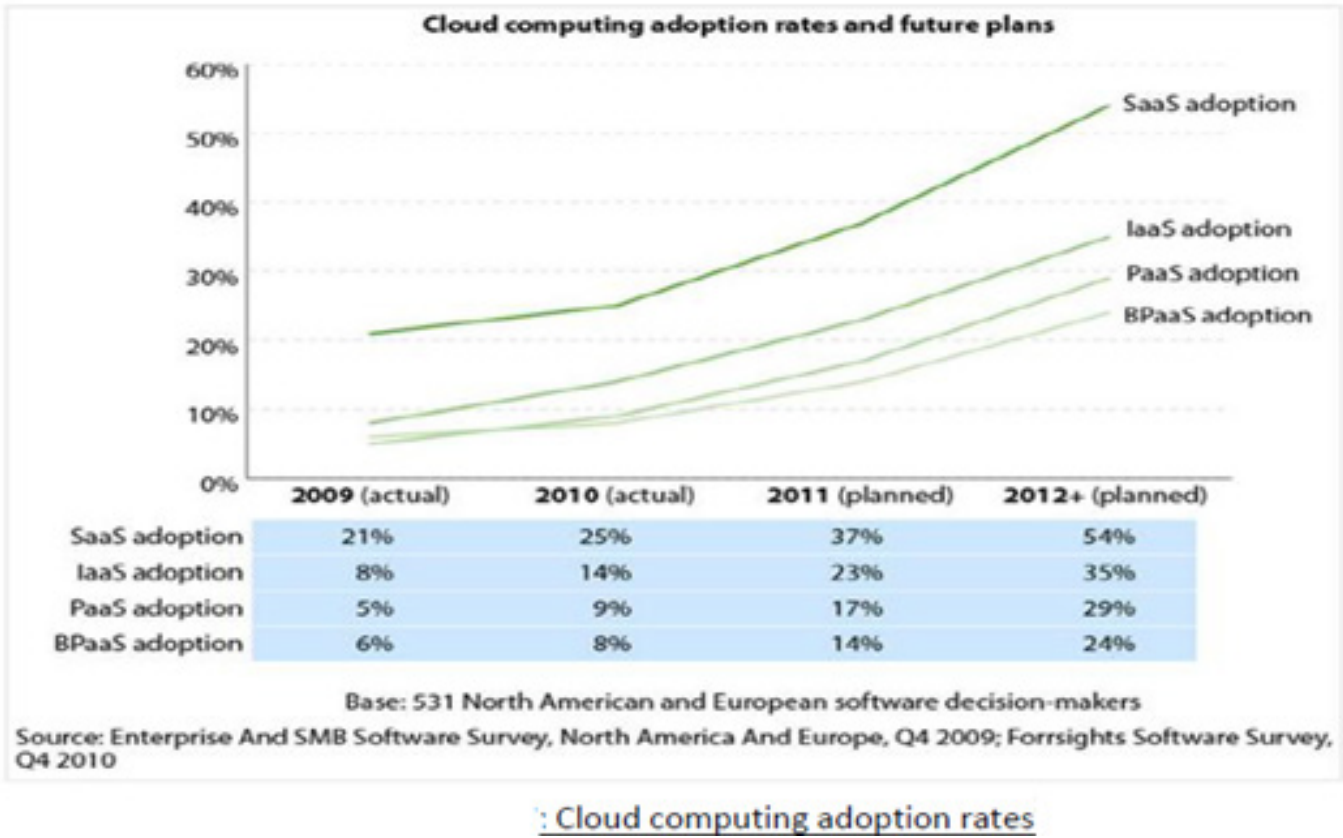

Figure 6. Cloud pomputing adoption rates and future plans

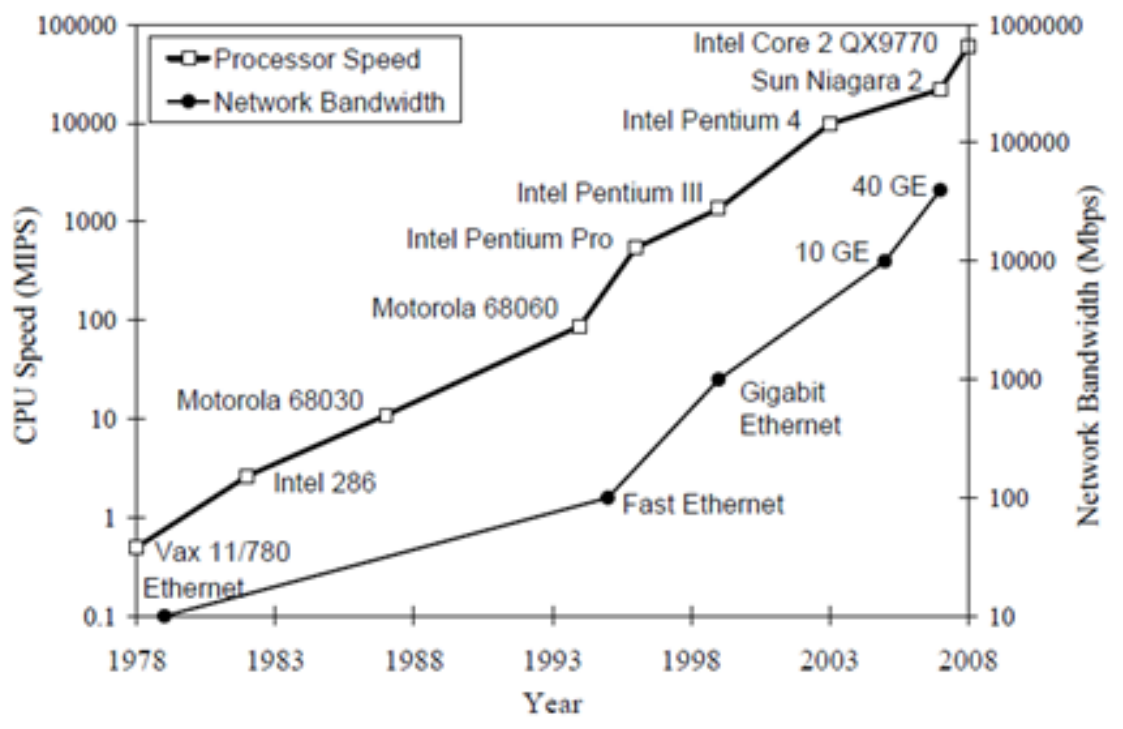

Improvement of processor and network technologies over 30 years.

Figure 7. Improvement of processor and network technologies over 30 years.

The rapid growth of flash memory and solid-state drive (SSD) also impacts the future of HPC and HTC systems. The mortality rate of SSD is not bad at all. A typical SSD can handle 300,000 -1,000,000 write cycles per block. So SSD can last for several years, even they have heavy write usage. Flash and SSD will demonstrate impressive speedups in many applications. For example, the Apple Macbook pro uses $128 \mathrm{~GB}$ solid-state hard drive, which is only $\$ 150$ more than a 500 GB 7200 RPM SATA drive. However to get 256 GB or 512 GB SSD drive, the cost may go up significantly. At present, SSD drives are still too expensive to replace stable disk arrays in the storage market. Eventually, power consumption, cooling and packaging will limit the large system development. The power increases linearly with respect to the clock frequency and quadratic ally with respect to the voltage applied on chips. We cannot increase the clock rate indefinitely. Lower the voltage supply is very much in demand. Today, wafer chip manufacturing technology is predominantly driven by planar metal oxide semiconductor field-effect transistor (MOSFET) technology. Advances in the areas of chip design and architecture have allowed semiconductor industries to reduce the size, density and cost of production of transistors. Technologies such as lithography, metrology and nanotechnology are used (and 
explored) to dramatically increase the number of transistors to be fabricated on a single chip. For example, semiconductor manufacturing processes have also improved from the current 32 nanometer $(\mathrm{nm})$ node in 2010 to the $22 \mathrm{~nm}$ node in late 2011 , moving to $16 \mathrm{~nm}$ by 2013 and $11 \mathrm{~nm}$ by 2015 (see Figure 3). Intel9, in April 2012, officially launched the world's first commercial microprocessor - "Ivy Bridge" on the 22nm wafer, using 3-D Tri-Gate technology. This $22 \mathrm{~nm}$ wafer is capable of fitting more than 2.9 billion transistors with $37 \%$ improvements in performance and more than $50 \%$ power reduction, compared to its predecessor transistors. Just as the size of the chips is getting smaller, the costs of sensing components are also dropping to become more affordable. Gartner has forecast that most technology components such as radio, WiFi, sensors and global positioning systems (GPS), could see a drop in cost of $15 \%$ to $45 \%$ from 2010 to 2015 (see Figure 4). To illustrate, with cheaper temperature sensors, cold chain retailers would consider deploying more temperature sensors to monitor their perishable produce as it traverses the supply chain. Excerpts from industry experts and practitioners reveal a raft of facets CC will possess come 2020 especially if the above challenges will have been sorted.

\section{Software Floats Away from Hardware}

John Manley, director of HP's Automated Infrastructure Lab, argues that software will become divorced from hardware, with more and more technologies consumed as a service: "Cloud computing is the final means by which computing becomes invisible," he says. As a result, by 2020, if you were to ask a CIO to draw a map of their infrastructure, they would not be able to, says David Merrill, chief economist of Hitachi Data Systems. "He will be able to say 'here are my partner providers'," he says, but he will not be able to draw a diagram of his infrastructure. This will be because it will be in a "highly abstracted space", where software is written in such a way that it goes through several filters before it interacts with hardware. This means that front-end applications, or applications built on top of a platform-as-a-service, will be hardware agnostic.

\section{Modular Software}

To take advantage of the huge armadas of hardware available via clouds, individual software applications are set to get larger and more complex as they are written to take advantage of scale. With the growth in the size and complexity of individual programs, the software development process will place an emphasis on modular software as in, large applications with components that can be modified without shutting down the program. As a consequence, cloud applications will require a new programming mindset, especially as they interact with multiple clouds. "Software has to be thought about differently," HP's Manley says, arguing that the management of federated services will be one of the main 2020 challenges. This is because applications are not only going to be based in the cloud, but will hook into other clouds and various on-premise applications as well. In other words, different parts of applications will "float around" in and out of service providers. Assuring good service-level agreements for these complex software packages will be a challenge, Manley says.

\section{Social Software}

Along with the modular shift, software could take on traits currently found in social-media applications like Face book, says Merrill. Programs could form automatic, if fleeting, associations with bits of hardware and software according to their needs. "It will be a social-media evolution," Merrill says. "You will have an infrastructure. It'll look like a cloud, but we will engineer these things so that a database will 'like' a server, [or] will 'like' a storage array." In other words, the infrastructure and software of a datacenter will mould itself around the task required, rather than the other way around. Developers will no longer need to worry about provisioning storage, a server and a switch, Merrill says: all of this will happen automatically.

\section{Commodity hardware rules}

By 2020 the transition to low-cost hardware will be in full swing as schemes such as the Open Compute Project find their way out of the datacenters of Face book and Amazon Web Services and into facilities operated by other, smaller companies as well. "Servers and storage devices will look like replaceable sleds," says Frank Frankovsky, Face book's VP of hardware design and supply chain, and chairman of the Open Compute Project.

"Cloud computing is the final means by which computing becomes invisible" - John Manley, HP. By breaking infrastructure down into its basic components, replacements and upgrades can be done quickly, he says. The companies best placed to use this form of commoditized infrastructure are large businesses that operate huge datacenters. "I would say that between now and 2020, the fastest-growing sector of the market is going to be cloud service providers," Frankovsky says.

\section{Low-power processors and cheaper clouds}

We're around a year away from low-power ARM chips coming to market with a 64-bit capability, and once that happens uptake should accelerate, as enterprise software will be developed for the RISC chips, allowing companies to use the power-thrifty processors in their datacenters, and thereby cut their electricity bills by an order of magnitude. HP has created a pilot server platform - Redstone - as part of its Project Moonshot scheme to try to get ARM kit to its customers, while Dell has been selling custom ARM-based servers to huge cloud customers via its Data Center Solutions group for years. By 2020 it's likely that low-power chips will be everywhere. And it won't just be ARM - Intel, aware of the threat, is working hard on driving down the power used by its Atom chips, though most efforts in this area are targeted at mobile devices rather than servers. Face book thinks ARM adoption is going to start in storage equipment, 
then broaden to servers. "I really do think it's going to have a dramatic impact on the amount of useful work, per dollar, you can get done," Frankovsky says. This should help cloud providers, such as Amazon Web Services, cut their electricity bills. Moreover, if they are caught in a price war with competitors, they are more likely to pass on at least a chunk of the savings to developers, in the form of price reductions.

\section{Faster Interconnects}

The twinned needs of massively distributed applications and a rise in the core count of high-end processors will converge to bring super-fast interconnects into the datacenter. Joseph Reger, chief technology officer of Fujitsu Technology Solutions, predicts that by 2020 we can expect communications in the datacenter to be "running at a speed in the low hundreds of gigabits per second". He says he expects that there will be a "very rapid commoditization" of high-end interconnect technologies, leading to a very cheap, very high-performance interconnect. This will let information be passed around datacenters at a greater rate than before, and at a lower cost, letting companies create larger applications that circulate more data through their hardware (known in the industry as 'chatty' apps), potentially allowing developers to build more intelligent, automated and complex programs.

\section{Datacenters Become Ecosystems}

Cloud datacenters will "become much like a breathing and living organism with different states", Reger says. The twinned technologies of abstracted software and commodified hardware should combine to make datacenters function much more like ecosystems, with over-arching system ruling equipment via software, with hardware controlled from a single point, but growing and shrinking according to workloads. Cloud datacenters will "become much like a breathing and living organism with different states". Automation of basic tasks, such as patching and updating equipment, will mean the datacenter "will become more like a biological system" he says, in the sense that changes and corrections are automatically made.

\section{Clouds Consolidate}

The internet rewards scale, and with the huge capital costs associated with running clouds, it seems likely that there will be a degree of consolidation in the cloud provider market. Fierce competition between a few large providers could be a good thing, as it would still drive each of them to experiment with radical technologies. For example, in a bid to cut its internal networking costs and boost utilization, Google has recently moved its entire internal network to the software-defined networking OpenFlow standard, which looks set to shake up the industry as more people adopt it. Manley of HP argues there will be a variety of clouds that will be suited to specific purposes. "There's going to be diversity," he says. "I think you would only end up with a monopoly if there was an infrastructure around that was sufficiently capable to meet all the non-functional (infrastructure requirements) of those end services."

\section{The Generational Shift}

By 2020, a new generation of CIOs will have come into companies, and they will have been raised in a cloudy as-a-service world. There will be an expectation that things are available "as-a-service", Merrill says: "Our consumption model is changing as a generational issue." And this new generation may lead to a shake-up in how businesses bill themselves for IT, Merrill says. "We have these archaic, tax-based, accounting-based rules that are prohibiting innovation," he adds.

\section{Clouds Will Stratify}

Today clouds are differentiated by whether they provide infrastructure-as-a-service, platform-as-a-service or software-as-a-service capabilities, but by 2020 more specialized clouds will have emerged. According to Forrester, we can expect things like 'middle virtualization tools' and 'dynamic BPO services' to appear by 2020 , along with a host of other inelegant acronyms. In other words, along with some large providers offering basic technologies like storage and compute, there will also be a broad ecosystem of more specific cloud providers, allowing companies to shift workloads to the cloud that would otherwise be dealt with by very specific (and typically very expensive) on-premise applications.

\section{The New Kind of Science(s)}

The long history of science can be divided into three significant periods: empirical, theoretical and experimental/simulation The first one was mainly experiential, without facilities to abstract, capture and share scientific knowledge. The second period of the great discoveries was followed by the formulation of the key scientific theories. They have been captured, shared and reused widely. We are currently passing the period of the great experimental sciences creating torrents of data used for simulation and an unprecedented level of sharing and collaboration among scientists. Evolving mega-structures are hosting the large scale social phenomena articulated as 'social computing'. There are suggestions to establish an entirely new type of scientific research based on the web fabrics called 'web science'. We believe that the emergence of cloud fabrics will enable new insights into challenging engineering, medical and social problems. Previously, we had no easy means to tackle peta-scale type of problems, nor carry out mega-scale simulation - which cloud computing should enable. One interesting thread will be to revisit 50 year old problems in Artificial Intelligence or explore further the 'Science of Services' - both being under the auspices of Complex Systems research.

In several ways the emergence of the computing cloud will invigorate academic research and will have strong potential to spawn innovative collaboration methods and new behaviors. Academic activities are well under way as 
the scientists have struggled for a long time with vast amounts of data coming not only from the web but also from a rising number of instruments and sensors. Cloud computing has particular strength as it draws on many existing technologies and architecture and integrates centralized, distributed and 'software as service' computing paradigms into an orchestrated whole. For now, C2 seems to be also a very promising computing technology paradigm. We may yet observe emergence of Academic Cloud Computing $(\mathrm{AC} 2)$ in the near future.

\section{Market Prognosis}

\section{Emergence of Lots of Clouds in the Sky}

Given the four forms of cloud service, there is ample room for hundreds or even thousands of players in the market. As such, there will be many "large clouds" and a lot more "small clouds". Many of the small clouds will live on some of the large clouds, that is, many small cloud computing vendors will offer their services on the utility computing services provided by large cloud computing vendors.

\section{Emergence of Cloud Integration Services}

Once the users start using services from multiple cloud service providers, the need will arise for migrating and integrating applications and data from different clouds. This will bring about a new form of cloud service, that is, cloud integration service. The integration technology will leverage such technologies as EAI (enterprise application integration), EII (enterprise information integration or federated database), and ESB (enterprise service bus).Formation of a cloud computing ecosystem. An ecosystem of cloud computing vendors will form. It will include various types of player, besides the vendors that offer cloud services to the users. One type of player already offers solutions that help enterprises to provision and manage virtual data centers from commodity servers and storage. They include 3Tera (AppLogic), Cohesive Flexible Technologies (ElasticServer on Demand), Liquid Computing (LiquidQ), Terremark, Layered Technologies, and Calibre[McLaughlin08][Gruman08]. A second type of player, such as Elastra and Maavo [Foley08], will offer solutions for deploying and managing applications in a data center (within an enterprise). Other types of player will include cloud computing platform vendors, the usual application software and middleware vendors, system integrators, and consultancies.

\section{Adoption of Hybrid Systems}

Because one cannot reasonably expect cloud computing to guarantee $100 \%$ availability and security, many users will adopt hybrid systems of clouds and on premises systems. The on-premises systems may include private clouds, that is, virtual data centers running within the firewall.

\section{Rising Subscription Fees}

The current low subscription fees are likely to go upwards significantly, as vendors harden their clouds (for higher performance, scalability, availability, and security), make their services richer, provide better support, and inevitably become profit-driven.

\section{Technical Intervention towards the Decade}

To help cloud computing take root, it will be necessary to adapt various mature technologies to the cloud computing paradigm listed below.

\section{Cloud Computing Software Platform}

As cloud computing software platform is the heart of a cloud computing system, it will require considerable further research. Hadoop is an open source cloud computing software platform, as an alternative to the platforms developed by Google and others. It appears to be a good vehicle as a launching point for research. Yahoo is a major sponsor of the Hadoop project. IBM has adopted Hadoop for its Blue Cloud solution. Face book uses Hadoop in its data analysis. Google, IBM, and Yahoo have donated cloud computing platforms to 6 US universities. The computing platforms all include Hadoop. Hadoop may become the Linux of cloud computing.

\section{Collaboration Applications}

Such means of collaboration as chat, instant messaging; Internet phone calling, etc. will be added to various popular applications. Google Docs spreadsheets already makes it possible for multiple users to chat while editing a spreadsheet together.

\section{Application And Data Integration Across Clouds}

The research on these subjects can leverage the available EAI, EII, and ESB technologies.

\section{Continuing Work On Multimedia Transmission and Data Mining}

Transmitting the bulky multimedia data across the network will continue to be a challenge, and it needs further research to speed up cloud computing. Further, as more data gets pushed to the clouds, including user-created data, the need to analyze (mine) such data to derive business-useful knowledge will increase. The data mining and machine learning communities will need to address this need.

\section{Service Management}

As the clouds proliferate and the users start plugging into multiple clouds, the problems of discovering and composing services that have been subjects of research in the service-oriented architecture context will need to be revisited in the cloud computing context. As cloud providers continue to utilize virtualization technologies in their systems, this can become problematic. In particular, the consolidation of multiple customer applications onto multicore servers introduces performance interference between collocated workloads, significantly impacting application QoS. 
From these analytic, summaries we can see that nanotechnologies are leading to molecular trends which have cognitive Behavioral characteristics and intelligence as in Figure 8

Power scavenging (energy harvesting) technologies that convert energy out of physical energy sources such as temperature differences and applied pressure, have been researched to explore their capability to replace conventional batteries. Two examples of power scavenging technologies are photovoltaic technology which generates electric power by using solar energy and piezoelectrics technology that creates charges on stress or shape change on voltage applied. Newer forms of battery technologies, e.g., polymer battery, fuel-cell and paper batteries will support increasing functionality and longer battery lifetime. Paper and smart label batteries have shown promising use cases in warehousing usage as they allow containers to perform two-way communications with the reader.

\section{Government Cloud Computing Markets 2015-2020}

Governments worldwide are increasingly deploying cloud computing technology. The global government cloud computing market is expected to grow at $6.7 \%$ CAGR generating \$118 Billion in revenues over 2015-2020 period which Figure 9 outlines as molecular nanosystem based.

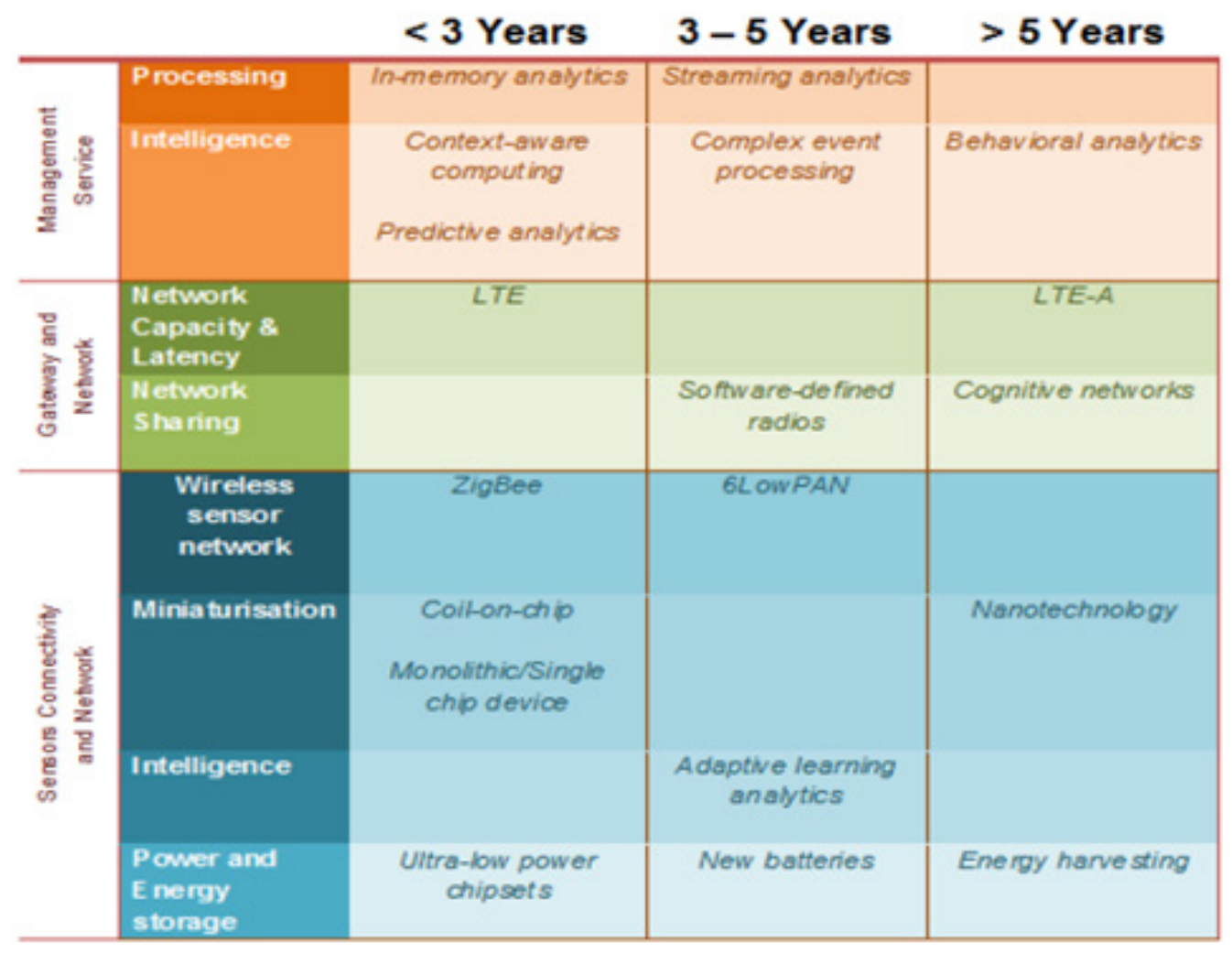

Figure 8. Technology Radar

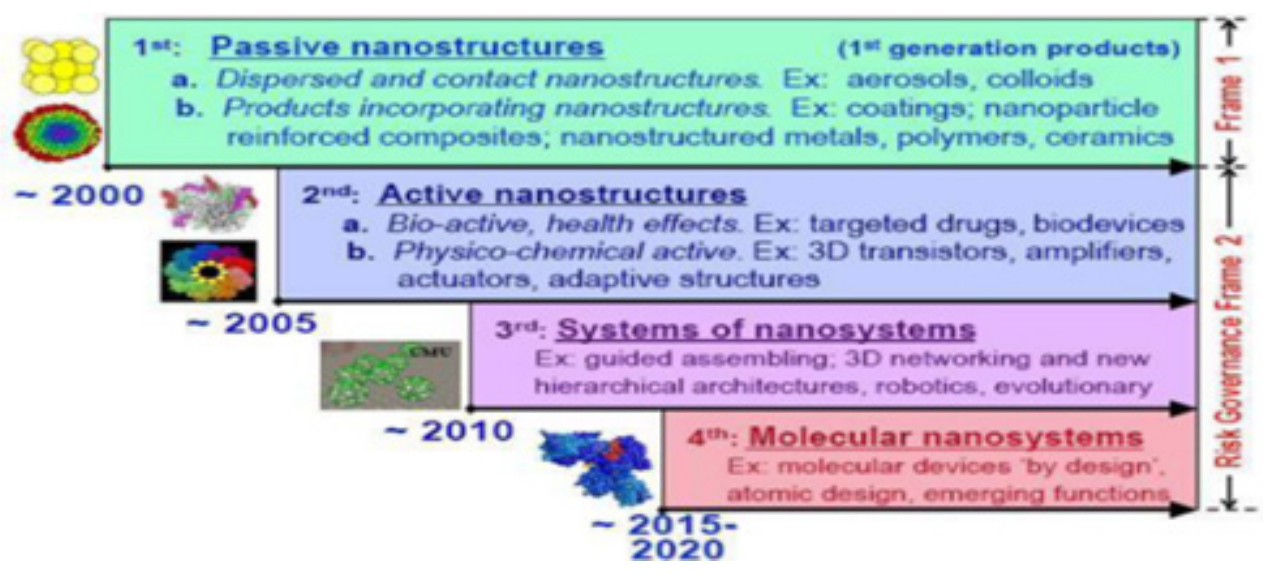

Figure 9. Nanocomputing Trajectory 


\section{Key Drivers For CC}

The world is accelerating into cloud computing. By around 2020, cloud service will be the primary IT consumption source for about 90 percent of individuals and enterprises. Key drivers of this future include:

-Even lower IT costs driven by higher

-Levels of automation and increased

- On-demand and self-service capabilities

-A revolution in the consumer supply chain

-Logistics enabled by new manufacturing

-Paradigms like digital product delivery and

three-dimensional printing

-Broadening network access allowing a global customer

base for nearly any product

-Cross-service provider resource pooling enabled by

inter-cloud standards and the ubiquitous use of cloud service broker

\section{Discussion}

As discussed above, various researchers have postulated the fact that nanotechnology is replacing semi conductor theory with molecular circuits an aspect which has lead to miniaturized devices with logic operations of remarkable complexity close to decision-making related to memory functions. This direction is likely to lead to development of "thinking" technologies in the near future which could greatly influence a complete change over of CC.

Independent research firm Forrester Research expects the global cloud computing market to reach $\$ 241$ billion in 2020 compared to $\$ 40.7$ in 2010 , according to a new Forrester report called "Sizing the Cloud". This is a growth that is much more than what has been witnessed in the past few years. Also, it is important to note that this survey reveals figures that are much more than what was predicted earlier, when the technology was new to the world. Out of all the sectors of cloud computing, it is believed that the maximum growth will occur in the IaaS sector. In fact, if recent research is to be believed, IaaS alone accounts for $41.3 \%$ of the compounded annual growth rate for this sector. This is predicted to be achieved by the year 2016.The increasing interest in IaaS or Infrastructure as a Service in the cloud is largely due to the enterprise and government growth in IT. Moreover, the provision of newer technologies in the cloud by IaaS providers makes the entire adaption more exciting, less expensive, and definitely more effective. Kirilov (2011) said such a rapid market growth is a double-edged sword in terms of business opportunities and average corporate performance within the cloud computing sector. Some enterprises will benefit from the booming cloud computing market and even larger number of start-ups and established enterprises will try to set foot in the SaaS, IaaS and PaaS segments. However, a vast number of companies are expected not to make their way to the top and close doors following the appearance of too many market players in a lucrative yet limited market for cloud computing services.
Only those that are able to adapt quickly to fast changing market conditions will be crowned victors and will grab the lion's share of a \$241 billion cloud computing market in 2020. According to Sizing the Cloud report, Software-as-a-Service (SaaS) offers more growth opportunity than any other segment in the still largely vague market for cloud computing services. SaaS will retain its position as a leading segment in cloud computing with SaaS market growing three-fold to $\$ 92.8$ billion by 2016 , the report said. In contrast, Infrastructure-as-a-service (IaaS) will witness a rapid growth in the next few years but Forrester expects dynamic infrastructure services to perform better than IaaS in the long term. Forrester applies an innovative approach toward measuring cloud computing market with company's analysts utilizing Forrester's sizing methodology for emerging markets to the cloud computing market for the first time, report co-author Stephen Reid wrote in a blog post. Reid admits that cloud computing definitions are still a serious challenge to researchers while each year, it gets harder to make clear-cut distinctions, based on technology, between what is a cloud and what is simply a virtualized data center or a traditional hosting provider. Therefore, Forrester decided to focus instead on the major disruption in the IT industry that cloud computing is bringing about - disruption based not on technology but on the business model under which IT operates." Reid also reveals that IaaS will shift from public clouds to virtual private clouds but the size of market is set to decrease because dynamic infrastructure services, which are the virtual private cloud counterpart of IaaS in the public cloud, tend to combine pure infrastructure with high-level services and close integration into existing on-premises landscapes. Simplified and pre-integrated platforms outline the future of Platform-as-a-service (PaaS) segment while PaaS is set to become a middleware platform alternative, Reid said. PaaS is a relatively small market now - about a tenth the size of Infrastructure as a Service (IaaS) - but it's doubling in size every year. By 2020, probably 80 percent of the new workloads will happen in the public cloud. Every time they face a new need, tomorrow's CIO will either pick a ready-to-consume SaaS if they can find one that matches their requirements or build an application on top of a PaaS, if they have custom needs a SaaS can't satisfy. In both cases, they won't care about what infrastructure is required to make that happen. This is what makes PaaS such an extremely strategic play: It will become the core tool of any enterprise developer. Kamthania et al (2009) talked of online operating systems and dumb terminalling which has come to pass in form of virtual desktop infrastructure (VDI) technologies championed by Dell. These are cheap and sometimes solar powered enabling easy access to the cloud. Enterprises who had over invested in PCs may have to dump them due to the emergence of desktop as a service through VDIs, Laird (2013).The blue cloud or federated clouds will have to compel practitioners who modeled their systems to run on single clouds to change and embrace cloud federation. Globalization and market integration will prefer $\mathrm{CC}$ which is 
market driven as Chang (2010) predicted as we can see e-commerce penetration already. Abas et all (2010) argument on educational $\mathrm{CC}$ and the impact of generation $\mathrm{Y}$ users who are the majority in the social media etc will need a more mobile and versatile platform hence applications currently running on rather static $\mathrm{CC}$ will have to re engineer. Molecular circuits will replace the solid state technology and result into super fast computing as stated in the literature review hence very mobile CC as reed et al (2009) predicted and this may impact great the informatics arena due to the "brainy" aspect of computing. Automation may lead to huge redundancies in terms of employment since traditional tasks like patching and upgrades will be automatic in the supersonic data centers which will form ecosystems with breathing organism like data centers with various states, Reger (2010).

\section{Conclusion}

The near future will definitely see a changed CC with different programming needs, fast and rather federation. Enterprises with applications in the clouds will have to re engineer them to suit the new architectures and dispose rigid ones of course with loss of investment and layoffs. Those out of the CC will have to join in since system compatibilities will depend on one membership in the CC. This is shown by the numerical evidence given above. As a recommendation, users are advised to consult widely and opt for more open and flexible technologies easier to change before taking up CC. The research fraternity is also called upon to gang with $\mathrm{CC}$ practitioners to develop seamless upgrades with limited possible disruption.

\section{REFERENCES}

[1] InterCloud: Utility-Oriented Federation of Cloud Computing Environments for Scaling of Application Services Buyya et al (2010)

[2] Conference Information, Vanessa Chang, Curtin University, Australia; Christian Guetl, Graz University of Technology, Austria

[3] Computing With Molecules, By Mark A. Reed and James M. Tour(2010)

[4] Jack Clark for Cloud Watch | August 1, 2012 -- 11:00 GMT (04:00 PDT)

[5] Cloud computing: today and tomorrow 70 journal of object technology vol. 8 , no. 1 Vol. 8 , no.1 journal of object technology 71

[6] Q-clouds: managing performance interference effects for QoS-aware clouds Nathuji et al

[7] Pages 237-250 ACM NewYork, NY,USA (C2010 table of contents ISBN:

[8] 978-1-60558-577-2doi>10.1145/1755913.1755938

[9] Berners-Lee T. et al, Creating a Science of the Web, Science, Vol. 313, 11 August 2006. pp. 769-771

[10] Delic K., On Dependability of Corporate Grids, ACM Ubiquity, Volume 6, Issue 45 (December 7-13, 2005)

[11] Delic K.,Walker M., Architecting Enterprise Grids: Possible Inflection Points, IADIS

[12] International Conference on Applied Computing, February

[13] 2007. Forrester Research Inc. Is Cloud Computing Ready for The Enterprise? March7, 2008

[14] Foster I., Service-Oriented Science, Science, Vol. 308, 6 May 2005. pp. 814-817 Hand E., Head in the clouds, Nature, Vol. 449, 24 October 2007, p. 963 Robison S., A Bright Future in the Cloud, Financial Times, March 4, 2008. Strong P., Enterprise Grid Computing, ACM Queue Magazine, July/August 2005. The Economist: When Clouds Collide, February 7, 2008.

[15] Weiss A., Computing in The Clouds, ACM netWorker, December 2007, pp. 16-25

[16] Next generation computing kamthania et al(2009)

[17] Molecular Logic Circuits $\dagger$ Vincenzo Balzani Prof., Alberto Credi Dr.,Margherita Venturi Prof.Article first published online: 30 DEC 2002 DOI: 10.1002/cphc.200390007 Advanced Molecular Logic with Memory Function† Uwe Pischel Dr. Article first published online: 25 JAN 2010 DOI: 10.1002/anie.200906705 Copyright C 2010 WILEY-VCH Verlag GmbH \& Co. KGaA, Weinheim

[18] Electronics using hybrid-molecular and mono-molecular devices C. Joachim1, J. K. Gimzewski2 \& A. Aviram3 Data Processing on a Unimolecular Platform†

[19] He Tian Article first published online: 11 JUN 2010 DOI: 10.1002/anie.200906834 Copyright (C) 2010 WILEY-VCH Verlag GmbH \& Co. KGaA, Weinheim

[20] Digital Processing and Communication with Molecular Switches F.M. Raymo Article first published online: 12 MAR 2002

DOI: 10.1002/1521-4095(20020318)14:6<401::AID-ADMA $401>3.0 . \mathrm{CO} ; 2-\mathrm{F}$

[21] Distributed Computing : Clusters, Grids and Clouds, All rights reserved by Kai Hwang, Geoffrey Fox, and Jack Dongarra, May 2, 2010.

[22] http://postscapes.com/cisco-internet-of-things-white-paper-h ow-the-next-evolution-of-the-internet-is-changing-everythin g\%20

[23] http://www.getcloudservices.com/blog/iaas-set-to-encourage -cloud-computing-growth

[24] http://www.cloudtweaks.com/2011/04/cloud-computing-mar ket-will-top-241-billion-in-2020/

[25] http://www.thinkgig.com/wp-content/uploads/2012/11/centu rylink-business-technology-2020-ebook.pdf

[26] http://venturebeat.com/2013/09/04/why-paas-isnt-just-for-sta rtups/\#4tSh3FtI7EoDXGuC.99 